\title{
Effective potentials in the integral quantum Hall effect
}

\author{
F. Lado* \\ Department of Physics, North Carolina State University, Raleigh, North Carolina 27695-8202, USA
}

(Received 29 September 2002; revised manuscript received 14 November 2002; published 26 June 2003)

\begin{abstract}
The exact $n$-body distribution functions are calculated for a two-dimensional, noninteracting quantum electron gas in an external magnetic field for any temperature and density. At low temperature and filled lowest Landau level, these functions are identical to the exact distribution functions obtained by Jancovici [Phys. Rev. Lett. 46, 386 (1981)] for the classical two-dimensional one-component plasma (2DOCP) at the special plasma parameter $\Gamma=2$. This establishes that the quantum state with filling factor $\nu=1$, associated with the integral quantum Hall effect, is precisely described by an effective classical potential $\phi(r)=-2 \ln r$, so that a classical Boltzmann factor of 2DOCP form can replace the quantum Slater sum. Further, this Boltzmann factor exactly matches that constructed by Laughlin [Phys. Rev. Lett. 50, 1395 (1983)] to account for the fractional quantum Hall effect. Additional effective potentials for higher filling factors $\nu=2,3, \ldots$ are obtained semianalytically from the exact Yvon-Born-Green integral equation and numerically from the approximate hypernetted-chain integral equation. They have the asymptotic form $\phi(r) \sim-(2 / \nu) \ln r$.
\end{abstract}

DOI: 10.1103/PhysRevB.67.245322

PACS number(s): 73.43.-f, 71.10.Ca, 61.20.Gy

\section{INTRODUCTION}

A system of noninteracting electrons in a plane subject to an external magnetic field, first studied by Landau ${ }^{1}$ in 1930 to account for diamagnetism, became the basis for understanding the integral quantum Hall effect, ${ }^{2}$ discovered by von Klitzing et al. ${ }^{3} 50$ years later. The discovery of the fractional quantum Hall effect ${ }^{4}$ not long after called for a different explanation and an important contribution was made by Laughlin, ${ }^{5,6}$ who invoked the classical two-dimensional onecomponent plasma ${ }^{7}$ in constructing his solution.

In this work, we report a surprising connection between the same two-dimensional one-component plasma (2DOCP) and the integral quantum Hall effect, as modeled by the simplest system, Landau's noninteracting electrons in a plane. The connection is made after calculating the exact $n$-body distribution functions of this system, carried out in Sec. II, and realizing that, in a regime of low temperature and Landau-level filling factor $\nu=1$, they are identical to the exact distribution functions of the 2DOCP found by Jancovici $^{8}$ for plasma parameter $\Gamma=2$. This establishes that the quantum state with filling factor $\nu=1$ is precisely described by an equivalent classical potential $\phi(r)=-2 \ln r$. Effective potentials that apply to the integral quantum Hall effect at larger filling factors $\nu=2,3, \ldots$ are then found semianalytically from the exact Yvon-Born-Green integral equation ${ }^{9}$ in Sec. III and numerically from the approximate hypernetted-chain integral equation ${ }^{9}$ in Sec. IV. They have the asymptotic form $\phi(r) \sim-(2 / \nu) \ln r$.

\section{GENERAL $\boldsymbol{n}$-PARTICLE DISTRIBUTION FUNCTION}

We consider $N$ electrons at temperature $T$ in area $A$. A uniform magnetic field $B_{0}$ is applied perpendicular to the plane of $A$. The canonical partition function $Q_{N}$ for this system can be written

$$
Q_{N}=\frac{1}{N ! \Lambda^{2 N}} \int W_{N}\left(\boldsymbol{r}^{N}\right) d \boldsymbol{r}_{1} \cdots d \boldsymbol{r}_{N},
$$

where $W_{N}\left(\boldsymbol{r}^{N}\right)$ is the (antisymmetrized) Slater sum,

$$
W_{N}\left(\boldsymbol{r}^{N}\right)=\Lambda^{2 N} \sum_{P}(-1)^{|P|} \sum_{k} \Psi_{k}^{*}\left(P \boldsymbol{r}^{N}\right) e^{-\beta \mathcal{H}} \Psi_{k}\left(\boldsymbol{r}^{N}\right),
$$

which plays the role of Boltzmann factor. The conventional introduction of the thermal de Broglie wavelength $\Lambda$ $=\left(h^{2} / 2 \pi m_{e} k_{B} T\right)^{1 / 2}$ in Eq. (1) serves to make the Slater sum dimensionless, but has no effect on the results reported below. Here, the $\Psi_{k}$ is a complete set of $N$-particle states distinguished by an index $k$ representing $2 N$ quantum numbers; the sum over permutations $P$, with even or odd parity $|P|$ and antisymmetric weighting, accounts for the Fermi-Dirac statistics of the indistinguishable electrons. The Hamiltonian $\mathcal{H}$ for noninteracting electrons in an external magnetic field is

$$
\mathcal{H}_{N}=\frac{1}{2 m_{e}} \sum_{j=1}^{N}\left[-i \hbar \nabla_{j}+e \boldsymbol{A}\left(\boldsymbol{r}_{j}\right)\right]^{2},
$$

where $m_{e}$ is the electron mass, $-\boldsymbol{e}$ is its charge, and $\boldsymbol{A}(\boldsymbol{r})$ is a vector potential that yields a uniform magnetic field $\boldsymbol{B}_{0}$ (which we shall take to define the $z$ direction),

$$
\boldsymbol{B}_{0}=B_{0} \hat{\boldsymbol{k}}=\boldsymbol{\nabla} \times \boldsymbol{A}(\boldsymbol{r}) .
$$

As usual, $\beta=1 / k_{B} T$ is the inverse temperature, with $k_{B}$ Boltzmann's constant. The system is taken to be fully spin polarized, so that spin degrees of freedom may be ignored.

Since interactions between electrons are being neglected, the Schrödinger equation for the energy eigenstates is readily solved and the sum over states in Eq. (2) can be carried out. The result is 


$$
\begin{aligned}
W_{N}\left(\boldsymbol{r}^{N}\right)= & \left(\Lambda^{2} / 4 \pi l_{0}^{2} \sinh \beta \mu_{B} B_{0}\right)^{N} \sum_{P}(-1)^{|P|} \\
& \times \exp \left\{-\sum_{j=1}^{N}\left[\left(\boldsymbol{r}_{j}-P \boldsymbol{r}_{j}\right)^{2} / 4 l_{0}^{2} \tanh \beta \mu_{B} B_{0}\right.\right. \\
& \left.\left.-i\left(x_{j} P y_{j}-y_{j} P x_{j}\right) / 2 l_{0}^{2}\right]\right\}
\end{aligned}
$$

where $\boldsymbol{r}_{j}$ locates electron $j$ in the $x y$ plane, $\mu_{B}=e \hbar / 2 m_{e}$ is the Bohr magneton, and $l_{0}=\left(\hbar / e B_{0}\right)^{1 / 2}$ is the characteristic magnetic length. Letting the external field $B_{0}$ vanish recovers the Slater sum of ideal fermions,

$$
\left.W_{N}\left(\boldsymbol{r}^{N}\right)\right\rfloor_{B_{0}=0}=\sum_{P}(-1)^{|P|} \exp \left[-\frac{\pi}{\Lambda^{2}} \sum_{j=1}^{N}\left(\boldsymbol{r}_{j}-P \boldsymbol{r}_{j}\right)^{2}\right] .
$$

A derivation of Eq. (5) using the asymmetric Landau gauge for $\boldsymbol{A}(\boldsymbol{r})$ is given in the Appendix.

With the Slater sum in hand, we seek to determine the $n$-body distribution function

$$
\rho_{N}^{(n)}\left(\boldsymbol{r}_{1}, \ldots, \boldsymbol{r}_{n}\right)=\frac{1}{(N-n) ! \Lambda^{2 N} Q_{N}} \int W_{N}\left(\boldsymbol{r}^{N}\right) d \boldsymbol{r}_{n+1} \cdots d \boldsymbol{r}_{N}
$$

for any $n$. One notes that each permutation $P$ in Eq. (5) produces a disjoint set of ring-type integrands, with each particle appearing in one and only one ring. Particle positions $1,2, \ldots, n$ are not integrated out and will be called root points. The remaining $N-n$ particle positions are variables of integration, to be called field points. The bond linking adjacent particles $i$ and $j$ in a ring is

$$
\begin{aligned}
f\left(\boldsymbol{r}_{i}, \boldsymbol{r}_{j}\right)= & \left(4 \pi l_{0}^{2} \sinh \beta \mu_{B} B_{0}\right)^{-1} \\
& \times \exp \left[-\left(\boldsymbol{r}_{i}-\boldsymbol{r}_{j}\right)^{2} / 4 l_{0}^{2} \tanh \beta \mu_{B} B_{0}\right. \\
& \left.+i\left(x_{i} y_{j}-y_{i} x_{j}\right) / 2 l_{0}^{2}\right] .
\end{aligned}
$$

Let $I_{k}\left(\boldsymbol{r}_{i}, \boldsymbol{r}_{j}\right)$ be a chain of links beginning at root point $\boldsymbol{r}_{i}$ and ending at root point $\boldsymbol{r}_{j}$ with $k$ field points in between, i.e.,

$$
I_{k}\left(\boldsymbol{r}_{i}, \boldsymbol{r}_{j}\right)=\int f\left(\boldsymbol{r}_{i}, \boldsymbol{r}_{n_{1}}\right) f\left(\boldsymbol{r}_{n_{1}}, \boldsymbol{r}_{n_{2}}\right) \cdots f\left(\boldsymbol{r}_{n_{k}}, \boldsymbol{r}_{j}\right) d \boldsymbol{r}_{n_{1}} \cdots d \boldsymbol{r}_{n_{k}} .
$$

The canonical distribution function $\rho_{N}^{(n)}\left(\boldsymbol{r}_{1}, \ldots, \boldsymbol{r}_{n}\right)$ then becomes ${ }^{10}$

$$
\begin{aligned}
\rho_{N}^{(n)}\left(\boldsymbol{r}_{1}, \ldots, \boldsymbol{r}_{n}\right)= & \frac{1}{(N-n) ! Q_{N}} \sum_{P}(-1)^{|P|} \sum_{m=0}^{N-n}(-1)^{m} \\
& \times \frac{(N-n) !}{(N-n-m) !} \sum_{k_{1}, k_{2}, \ldots, k_{n}} \delta\left(m, \sum_{j} k_{j}\right) \\
& \times I_{k_{1}}\left(\boldsymbol{r}_{1}, P \boldsymbol{r}_{1}\right) I_{k_{2}}\left(\boldsymbol{r}_{2}, P \boldsymbol{r}_{2}\right) \cdots I_{k_{n}}\left(\boldsymbol{r}_{n}, P \boldsymbol{r}_{n}\right) \\
& \times(N-n-m) ! Q_{N-n-m} .
\end{aligned}
$$

Now the permutations $P$ are among the $n$ root points only, while $m$ is the total number of field points in the rooted rings. The sums over number of field points $k_{j}$ in individual chains are collectively constrained by the requirement $\sum_{j=1}^{n} k_{j}=m$, expressed by the Kronecker delta $\delta(i, j)$ in the summand. This constraint can be removed, and the individual sums over the $k_{j}$ factored, by transforming these expressions from particle number $N$ to chemical potential $\mu$ as an independent variable using the grand canonical formalism. We have then

$$
\Xi=\sum_{N=0}^{\infty} e^{\beta \mu N} Q_{N}
$$

for the partition function and

$$
\rho^{(n)}\left(\boldsymbol{r}_{1}, \ldots, \boldsymbol{r}_{n}\right)=\Xi^{-1} \sum_{N=n}^{\infty} e^{\beta \mu N} Q_{N} \rho_{N}^{(n)}\left(\boldsymbol{r}_{1}, \ldots, \boldsymbol{r}_{n}\right)
$$

for the $n$-body distribution function. Using Eq. (10) in the latter, we now get simply

$$
\begin{aligned}
& \rho^{(n)}\left(\boldsymbol{r}_{1}, \ldots, \boldsymbol{r}_{n}\right) \\
& \quad=\sum_{P}(-1)^{|P|} \prod_{j=1}^{n}\left[\sum_{k=0}^{\infty}(-1)^{k} e^{(k+1) \beta \mu} I_{k}\left(\boldsymbol{r}_{j}, P \boldsymbol{r}_{j}\right)\right] .
\end{aligned}
$$

Starting from $I_{0}\left(\boldsymbol{r}_{i}, \boldsymbol{r}_{j}\right)=f\left(\boldsymbol{r}_{i}, \boldsymbol{r}_{j}\right)$, one finds by induction that

$$
\begin{aligned}
I_{k}\left(\boldsymbol{r}_{i}, \boldsymbol{r}_{j}\right)= & {\left[4 \pi l_{0}^{2} \sinh \left((k+1) \beta \mu_{B} B_{0}\right)\right]^{-1} } \\
& \times \exp \left[-\left(\boldsymbol{r}_{i}-\boldsymbol{r}_{j}\right)^{2} / 4 l_{0}^{2} \tanh \left((k+1) \beta \mu_{B} B_{0}\right)\right. \\
& \left.+i\left(x_{i} y_{j}-y_{i} x_{j}\right) / 2 l_{0}^{2}\right]
\end{aligned}
$$

so that finally,

$$
\begin{aligned}
\rho^{(n)}\left(\boldsymbol{r}_{1}, \ldots, \boldsymbol{r}_{n}\right)= & \sum_{P}(-1)^{|P|} \prod_{j=1}^{n}\left\{\eta\left(\left|\boldsymbol{r}_{j}-P \boldsymbol{r}_{j}\right|\right)\right. \\
& \left.\times \exp \left[i\left(x_{j} P y_{j}-y_{j} P x_{j}\right) / 2 l_{0}^{2}\right]\right\},
\end{aligned}
$$

with

$$
\begin{aligned}
\eta(r)= & \frac{1}{4 \pi l_{0}^{2}} \sum_{j=1}^{\infty} \frac{(-1)^{j-1} e^{j \beta \mu}}{\sinh \left(j \beta \mu_{B} B_{0}\right)} \\
& \times \exp \left[-r^{2} / 4 l_{0}^{2} \tanh \left(j \beta \mu_{B} B_{0}\right)\right] .
\end{aligned}
$$

This key result can be rewritten in a form that more directly displays the familiar Fermi degeneracy at low temperatures. We use the generating function of Laguerre polynomials,

$$
\frac{e^{-x z /(1-z)}}{1-z}=\sum_{k=0}^{\infty} L_{k}(x) z^{k}
$$

with $x=r^{2} / 2 l_{0}^{2}$ and $z=\exp \left(-2 j \beta \mu_{B} B_{0}\right)$ in Eq. (16) and then sum over $j$ to get 


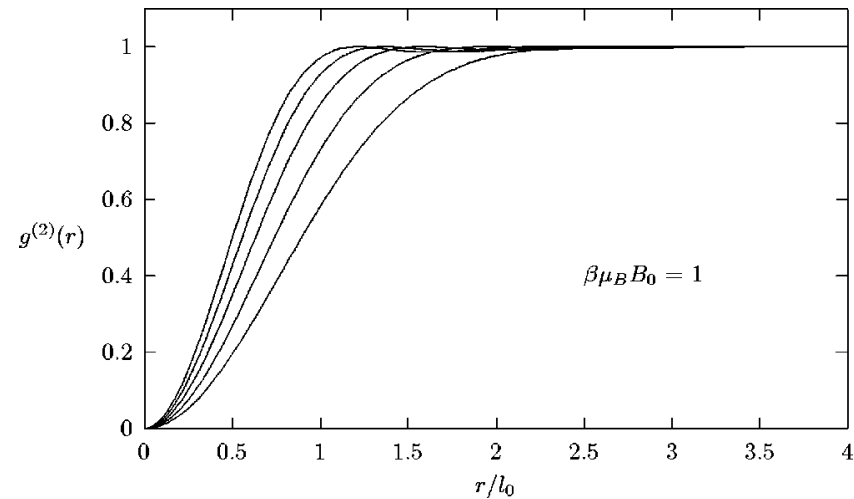

FIG. 1. Pair distribution functions of a two-dimensional, noninteracting quantum electron gas in a magnetic field at a high temperature and reduced densities $2 \pi \rho l_{0}^{2}=1,2,3,4,5$, read from right to left.

$$
\eta(r)=\frac{e^{-r^{2} / 4 l_{0}^{2}}}{2 \pi l_{0}^{2}} \sum_{k=0}^{\infty} \frac{L_{k}\left(r^{2} / 2 l_{0}^{2}\right)}{1+e^{-\beta\left[\mu-(2 k+1) \mu_{B} B_{0}\right]}},
$$

where now the summation is over Landau levels, of energy $\epsilon_{k}=(2 k+1) \mu_{B} B_{0}$.

The first of the distribution functions (15),

$$
\rho^{(1)}\left(\boldsymbol{r}_{1}\right)=\eta(0),
$$

is just the uniform density $\rho=\bar{N} / A$ expressed in terms of the chemical potential $\mu$,

$$
\rho=\frac{1}{2 \pi l_{0}^{2}} \sum_{k=0}^{\infty} \frac{1}{1+e^{-\beta\left[\mu-(2 k+1) \mu_{B} B_{0}\right]}} .
$$

This result is more familiarly obtained directly from the partition function $\Xi$, where

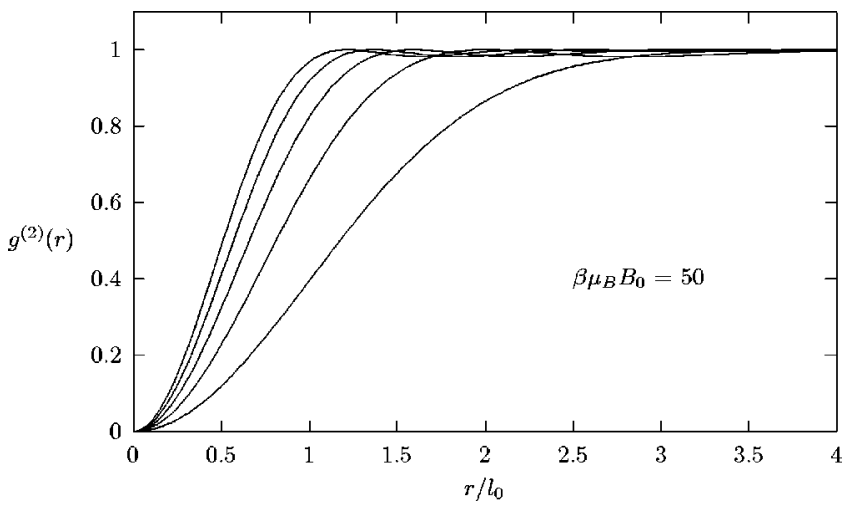

FIG. 2. Pair distribution functions of a two-dimensional, noninteracting quantum electron gas in a magnetic field at a low temperature and reduced densities $2 \pi \rho l_{0}^{2}=1,2,3,4,5$, read from right to left.

$$
\ln \Xi=\frac{A}{2 \pi l_{0}^{2}} \sum_{k=0}^{\infty} \ln \left\{1+e^{\beta\left[\mu-(2 k+1) \mu_{B} B_{0}\right]}\right\},
$$

using the thermodynamic relation $\bar{N}=k_{B} T \partial \ln \Xi / \partial \mu$.

The dimensionless forms

$$
g^{(n)}\left(\boldsymbol{r}_{1}, \ldots, \boldsymbol{r}_{n}\right) \equiv \rho^{(n)}\left(\boldsymbol{r}_{1}, \ldots, \boldsymbol{r}_{n}\right) / \rho^{n}
$$

are preferable in writing out higher distribution functions. Explicitly, we have for the next three,

$$
\begin{aligned}
& g^{(2)}\left(\boldsymbol{r}_{1}, \boldsymbol{r}_{2}\right)=1-D^{2}\left(r_{12}\right), \\
& g^{(3)}\left(\boldsymbol{r}_{1}, \boldsymbol{r}_{2}, \boldsymbol{r}_{3}\right)=1-\left[D^{2}\left(r_{12}\right)+D^{2}\left(r_{13}\right)+D^{2}\left(r_{23}\right)\right] \\
& +2 D\left(r_{12}\right) D\left(r_{23}\right) D\left(r_{31}\right) \\
& \times \cos \left(\left|\boldsymbol{r}_{12} \times \boldsymbol{r}_{13}\right| / 2 l_{0}^{2}\right) \text {, }
\end{aligned}
$$

$$
\begin{aligned}
g^{(4)}\left(\boldsymbol{r}_{1}, \boldsymbol{r}_{2}, \boldsymbol{r}_{3}, \boldsymbol{r}_{4}\right)= & 1-\left[D^{2}\left(r_{12}\right)+D^{2}\left(r_{13}\right)+D^{2}\left(r_{14}\right)+D^{2}\left(r_{23}\right)+D^{2}\left(r_{24}\right)+D^{2}\left(r_{34}\right)\right] \\
& +\left[D^{2}\left(r_{12}\right) D^{2}\left(r_{34}\right)+D^{2}\left(r_{13}\right) D^{2}\left(r_{24}\right)+D^{2}\left(r_{14}\right) D^{2}\left(r_{23}\right)\right] \\
& +2\left[D\left(r_{12}\right) D\left(r_{23}\right) D\left(r_{31}\right) \cos \left(\left|\boldsymbol{r}_{12} \times \boldsymbol{r}_{13}\right| / 2 l_{0}^{2}\right)+D\left(r_{13}\right) D\left(r_{34}\right) D\left(r_{41}\right) \cos \left(\left|\boldsymbol{r}_{13} \times \boldsymbol{r}_{14}\right| / 2 l_{0}^{2}\right)\right. \\
& \left.+D\left(r_{12}\right) D\left(r_{24}\right) D\left(r_{41}\right) \cos \left(\left|\boldsymbol{r}_{12} \times \boldsymbol{r}_{14}\right| / 2 l_{0}^{2}\right)+D\left(r_{23}\right) D\left(r_{34}\right) D\left(r_{42}\right) \cos \left(\left|\boldsymbol{r}_{23} \times \boldsymbol{r}_{24}\right| / 2 l_{0}^{2}\right)\right] \\
& -2\left[D\left(r_{12}\right) D\left(r_{23}\right) D\left(r_{34}\right) D\left(r_{41}\right) \cos \left(\left|\boldsymbol{r}_{13} \times \boldsymbol{r}_{24}\right| / 2 l_{0}^{2}\right)+D\left(r_{12}\right) D\left(r_{24}\right) D\left(r_{43}\right) D\left(r_{31}\right) \cos \left(\left|\boldsymbol{r}_{14} \times \boldsymbol{r}_{23}\right| / 2 l_{0}^{2}\right)\right. \\
& \left.+D\left(r_{13}\right) D\left(r_{32}\right) D\left(r_{24}\right) D\left(r_{41}\right) \cos \left(\left|\boldsymbol{r}_{12} \times \boldsymbol{r}_{34}\right| / 2 l_{0}^{2}\right)\right],
\end{aligned}
$$

where $D(r)=\eta(r) / \eta(0)$. The three-body distribution function is the first to show correlations due to phase.

For low temperatures and large magnetic fields, i.e., for $\beta \mu_{B} B_{0} \gg 1$, the summand in Eq. (20) acts like a discrete unit-step function, so that for (integer) $n$ filled Landau levels one has $2 \pi \rho l_{0}^{2}=n$; similarly, using Eq. (18), the pair distribution function $g^{(2)}\left(r_{12}\right)=g^{(2)}\left(\boldsymbol{r}_{1}, \boldsymbol{r}_{2}\right)$ simplifies in this limit to

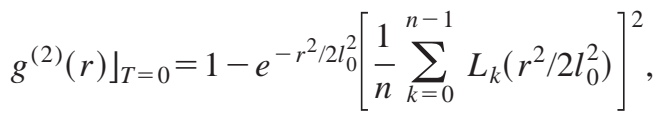

a result that has been obtained by Ciftja and Fantoni ${ }^{11,12}$ and Kamilla et al. ${ }^{13}$

More generally, the pair function $g^{(2)}(r)$ from Eq. (23) is calculated for density $\rho$ and arbitrary temperature $T$ from 


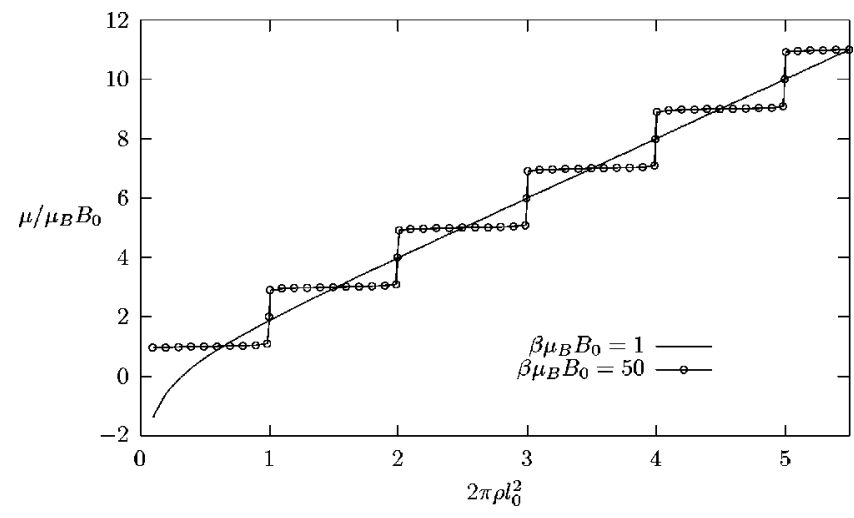

FIG. 3. Chemical potential as a function of density calculated by numerical inversion of Eq. (20) at high and low temperatures.

$$
g^{(2)}(r)=1-e^{-r^{2} / 2 l_{0}^{2}}\left[\frac{1}{2 \pi \rho l_{0}^{2}} \sum_{k=0}^{\infty} \frac{L_{k}\left(r^{2} / 2 l_{0}^{2}\right)}{1+e^{-\beta\left[\mu-(2 k+1) \mu_{B} B_{0}\right]}}\right]^{2},
$$

with chemical potential $\mu$ found by numerical inversion of Eq. (20). The controlling free parameter is then $\beta \mu_{B} B_{0}$. Figure 1 displays $g^{(2)}(r)$ for reduced densities $2 \pi \rho l_{0}^{2}$ $=1,2,3,4,5$ at $\beta \mu_{B} B_{0}=1$, while Fig. 2 shows the same functions at $\beta \mu_{B} B_{0}=50$. The latter are clearly longer ranged and have more structure (though neither set has much). For reference, Fig. 3 shows $\mu=\mu(\rho)$ from Eq. (20) for the two parameter values. Note that at low temperatures, the chemical potential computed at a discontinuity falls midway between its upper and lower limits: $\mu / \mu_{B} B_{0}=2 n$ when $2 \pi \rho l_{0}^{2}=n$ for $\beta \mu_{B} B_{0} \gg 1$.

The structure factors $S(k)=1+\rho \widetilde{h}(k)$ for the noninteracting electrons are now readily obtained by the Fourier transformation of $h(r)=g^{(2)}(r)-1$. For circularly symmetric functions such as these, the two-dimensional Fourier transform $\tilde{h}(k)$ and its inverse $h(r)$ become Hankel transforms,

$$
\begin{aligned}
& \tilde{h}(k)=2 \pi \int_{0}^{\infty} d r r h(r) J_{0}(k r), \\
& h(r)=\frac{1}{2 \pi} \int_{0}^{\infty} d k k \tilde{h}(k) J_{0}(k r),
\end{aligned}
$$

where $J_{0}(x)$ is the Bessel function of order zero. Structure factors for the pair distribution functions at $\beta \mu_{B} B_{0}=1$ and 50 that appear in Figs. 1 and 2 are displayed, respectively, in Figs. 4 and 5. The vanishing of $S(0)$ for all densities at low temperatures, seen in Fig. 5, signals the incompressibility of the quantum states with the filled Landau levels. The quadratures needed here for the transforms $\widetilde{h}(k)$ are performed numerically as described in Sec. IV.

We close this section by noting a remarkable fact. For physical conditions such that this noninteracting quantum electron system just fills the lowest Landau level, i.e., for $\beta \mu_{B} B_{0} \gg 1$ and $2 \pi \rho l_{0}^{2}=1$, so that $D(r)=\exp \left(-\pi \rho r^{2} / 2\right)$, the exact distribution functions $g^{(n)}\left(\boldsymbol{r}_{1}, \ldots, \boldsymbol{r}_{n}\right)$ displayed above are identical to the exact distribution functions

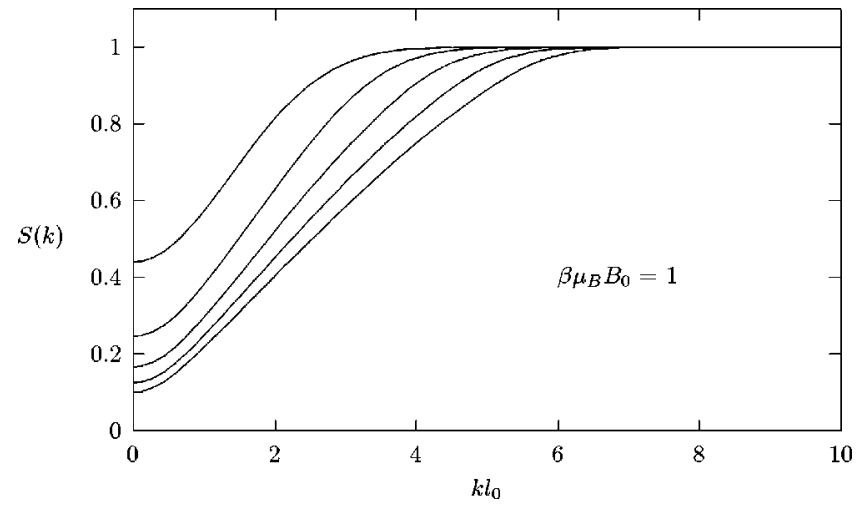

FIG. 4. Structure factors $S(k)=1+\rho \widetilde{h}(k)$ of a two-dimensional, noninteracting quantum electron gas in a magnetic field at a high temperature and reduced densities $2 \pi \rho l_{0}^{2}=1,2,3,4,5$, read from left to right.

$g^{(n)}\left(\boldsymbol{r}_{1}, \ldots, \boldsymbol{r}_{n}\right)$ of a classical two-dimensional onecomponent plasma obtained by Jancovici ${ }^{8}$ for the plasma parameter value $\Gamma=2$. Working backwards, we may then conclude that the quantum state for the filled lowest Landau level is precisely described by an effective classical pair potential $\phi(r)=-2 \ln \left(r / l_{0}\right)$. More generally, in the following section, we calculate effective potentials for a lowtemperature system with $n>1$ Landau levels filled and find that their long-range form is $\phi(r) \sim-(2 / n) \ln \left(r / l_{0}\right)$.

\section{AN EFFECTIVE PAIR POTENTIAL}

The Fermi statistics result in a well-known yet still curious "statistical repulsion" between noninteracting identical particles, as is evident in Figs. 1 and 2. The mathematical expression of this property through antisymmetrization of the wave function, however, often makes it awkward to work with. An explicit effective interaction between the fermions, which could produce the same effects as antisymmetrization, would considerably simplify the situation. This notion was first discussed by Uhlenbeck and Gropper, ${ }^{14,15}$ who carried out calculations for quantum gases in three dimensions at

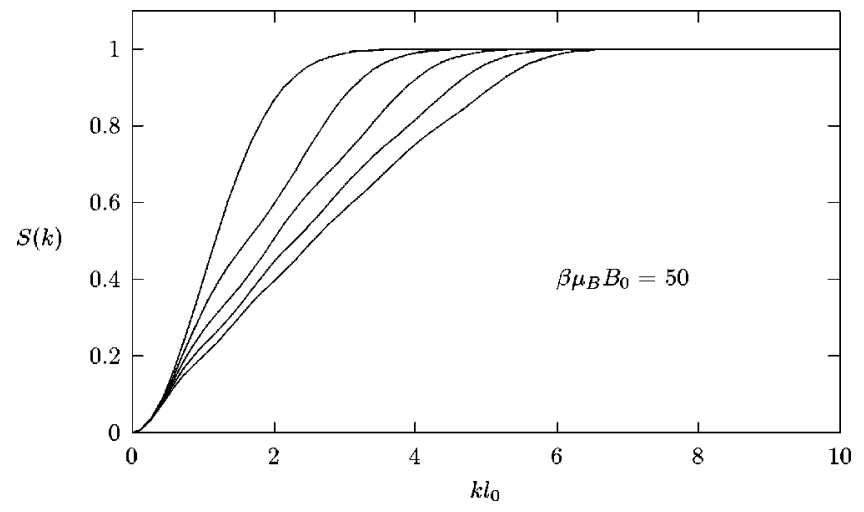

FIG. 5. Structure factors $S(k)=1+\rho \tilde{h}(k)$ of a two-dimensional, noninteracting quantum electron gas in a magnetic field at a low temperature and reduced densities $2 \pi \rho l_{0}^{2}=1,2,3,4,5$, read from left to right. The vanishing of $S(0)$ for all densities signals the onset of the effective 2DOCP interaction. 
high temperatures and low densities. A more general procedure suitable for any temperature and density, based on the exact Yvon-Born-Green hierarchy (YBG), was proposed by the author ${ }^{10}$ for the quantum gases with no external magnetic field. Stevens and Pokrant ${ }^{16}$ later noted that other familiar integral equations of classical liquids, ${ }^{9}$ such as PercusYevick and hypernetted-chain (HNC), though approximate, could also be used for this purpose. In this section and the next, we examine the extension of these techniques to the present case of a two-dimensional electron gas in a magnetic field.

The surprising conclusion of the preceding section is that the quantum correlation effects of antisymmetrization of the wave function can indeed be replicated by an effective classical pair potential for at least the case of filled lowest Landau level, $\nu=2 \pi \rho l_{0}^{2}=1$, at low temperatures. We continue here by assuming that there exists, for any state, a pair function $\phi(r)$ such that the Slater sum, Eq. (2), may be written

$$
W_{N}\left(\boldsymbol{r}^{N}\right) \approx \exp \left[-\sum_{i<j} \phi\left(r_{i j}\right)\right]
$$

Given that assumption, the YBG hierarchy ${ }^{9}$ then offers an exact route to such a function. The second member of this hierarchy, connecting the two-body and three-body distribution functions, reads

$$
\begin{aligned}
\boldsymbol{\nabla}_{1} \ln g^{(2)}\left(\boldsymbol{r}_{1}, \boldsymbol{r}_{2}\right)= & -\nabla_{1} \phi\left(r_{12}\right) \\
& -\rho \int d \boldsymbol{r}_{3} \boldsymbol{\nabla}_{1} \phi\left(r_{13}\right) \frac{g^{(3)}\left(\boldsymbol{r}_{1}, \boldsymbol{r}_{2}, \boldsymbol{r}_{3}\right)}{g^{(2)}\left(\boldsymbol{r}_{1}, \boldsymbol{r}_{2}\right)},
\end{aligned}
$$

where now $\phi(r)$ is the only unknown. Dotting $\hat{\boldsymbol{r}}_{12}$ into Eq. (31) gives a scalar equation,

$$
\begin{aligned}
-\frac{d \phi(r)}{d r}= & \frac{d \ln g(r)}{d r}-\frac{\rho}{g(r)} \int d s \cos \theta \frac{d \phi(s)}{d s} \\
& \times\left[D^{2}(t)-2 D(r) D(s) D(t) \cos \left(\frac{r s}{2 l_{0}^{2}} \sin \theta\right)\right],
\end{aligned}
$$

where $\cos \theta=\hat{\boldsymbol{r}} \cdot \hat{\boldsymbol{s}}, \quad t=\left(r^{2}+s^{2}-2 r s \cos \theta\right)^{1 / 2}$, and we have dropped those terms of $g^{(3)}\left(\boldsymbol{r}_{1}, \boldsymbol{r}_{2}, \boldsymbol{r}_{3}\right)$ that do not contribute because of symmetry. The superscript on the pair function is also omitted for simplicity. Then, with the substitutions

$$
\begin{aligned}
& u(r)=-\frac{d \phi(r)}{d r}, \\
& v(r)=\frac{d \ln g(r)}{d r},
\end{aligned}
$$

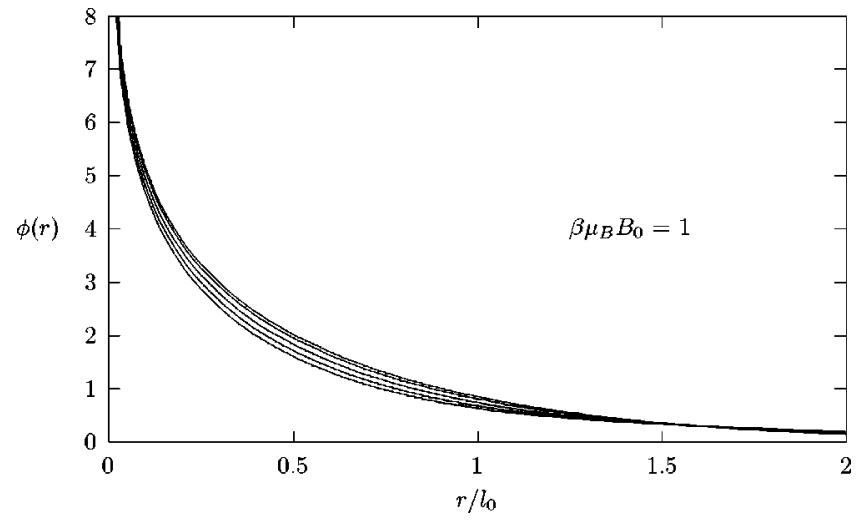

FIG. 6. Effective potentials obtained by numerical solution of the YBG equation for a two-dimensional, noninteracting quantum electron gas in a magnetic field at a high temperature and reduced densities $2 \pi \rho l_{0}^{2}=1,2,3,4,5$, read from top to bottom.

$$
\begin{aligned}
W(r, s)= & \frac{1}{\pi} \int_{0}^{\pi} d \theta \cos \theta\left[D^{2}(t)\right. \\
& \left.-2 D(r) D(s) D(t) \cos \left(\frac{r s}{2 l_{0}^{2}} \sin \theta\right)\right],
\end{aligned}
$$

Eq. (32) becomes an integral equation

$$
u(r)=v(r)+\frac{2 \pi \rho}{g(r)} \int_{0}^{\infty} d s s u(s) W(r, s)
$$

that can be solved iteratively for $u(r)$; a final integration

$$
\phi(r)=\int_{r}^{\infty} d s u(s)
$$

yields the effective potential. The integral for the array $W(r, s)$ in Eq. (35) is evaluated using the Gauss-Chebyshev quadrature.

For the high-temperature states displayed in Fig. 1, this straightforward procedure works well. The effective potentials found in this way for the states of Fig. 1 at $\beta \mu_{B} B_{0}$ $=1$ are shown in Fig. 6 . As the density increases, the amplitude of the effective potential decreases, but the changes are not large.

When applied at low temperatures, however, the simple procedures, both here and in the HNC solutions of the following section, become problematical as divergences make an appearance. Their source is not hard to find. (The isothermal compressibility $\chi_{T}$, given exactly by

$$
\rho k_{B} T \chi_{T}=\frac{1}{2 \pi \rho l_{0}^{2}} \sum_{k=0}^{\infty} \frac{e^{-\beta\left[\mu-(2 k+1) \mu_{B} B_{0}\right]}}{\left(1+e^{-\beta\left[\mu-(2 k+1) \mu_{B} B_{0}\right]}\right)^{2}},
$$

is relevant here and is plotted in Fig. 7.) Expanding the Bessel function $J_{0}(k r)$ in Eq. (28) and evaluating term by term, we get

$$
\rho \widetilde{h}(k)=-1+\frac{1}{2}\left(k l_{0}\right)^{2}+\cdots
$$




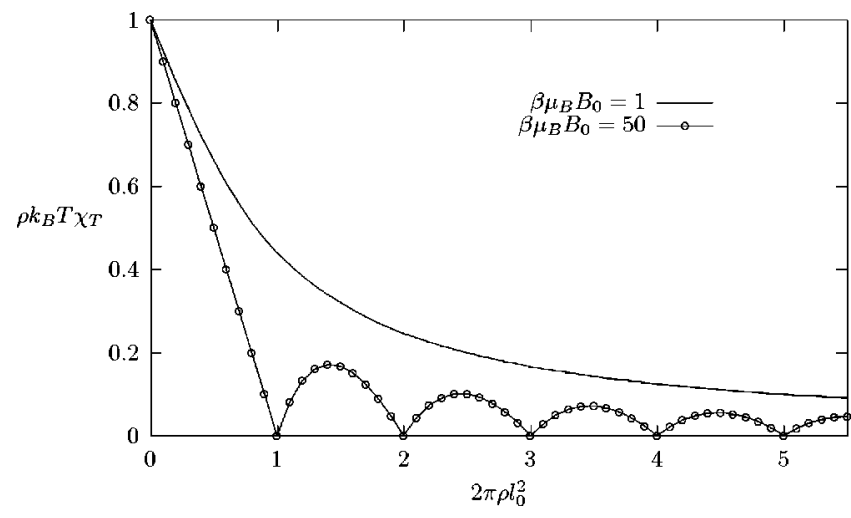

FIG. 7. Isothermal compressibility $\chi_{T}$ at high and low temperatures, calculated from Eq. (38). The vanishing of $\chi_{T}$ at low temperature for integral values of the reduced density $2 \pi \rho l_{0}^{2}$ is linked to $S(0)=0$ in Fig. 5 .

for $\beta \mu_{B} B_{0} \gg 1$ and $2 \pi \rho l_{0}^{2}=n$, physical conditions that will hold for the remaining section. Then, $S(0)=1+\rho \widetilde{h}(0)=0$, the compressibility $\chi_{T}=\beta S(0) / \rho$ vanishes, and the transform of the direct correlation function $c(r),{ }^{9}$

$$
\rho \tilde{c}(k)=\frac{\rho \widetilde{h}(k)}{1+\rho \widetilde{h}(k)},
$$

acquires a $k^{-2}$ divergence at the origin. The long-range behavior of $\phi(r)$ follows that of $-c(r)$ and so $\rho \widetilde{\phi}(k)$ $\approx 2 /\left(k l_{0}\right)^{2}$ for a small $k$, independent of density, and we finally get the asymptotic forms

$$
\begin{gathered}
\phi(r) \sim-(2 / n) \ln \left(r / l_{0}\right), \\
u(r) \sim 2 / n r .
\end{gathered}
$$

These are troublesome functions for any numerical procedure restricted to a finite range. Fortunately, it turns out that, in this low-temperature regime, many needed integrals can be evaluated analytically. Thus, using

$$
\begin{aligned}
& D(r)=\frac{1}{n} e^{-r^{2} / 4 l_{0}^{2}} \sum_{k=0}^{n-1} L_{k}\left(\frac{r^{2}}{2 l_{0}^{2}}\right), \\
& =\frac{1}{n} e^{-r^{2} / 4 l_{0}^{2}} L_{n-1}^{1}\left(\frac{r^{2}}{2 l_{0}^{2}}\right),
\end{aligned}
$$

we find, for example,

$$
W(r, s)=e^{-\left(\hat{r}^{2}+\hat{s}^{2}\right) / 2}\left[2 I_{1}(\hat{r} \hat{s})-\hat{r} \hat{s}\right] / 2
$$

for $n=1$,

$$
\begin{aligned}
W(r, s)= & e^{-\left(\hat{r}^{2}+\hat{s}^{2}\right) / 2}\left[3 2 \left(8-4 \hat{r}^{2}-4 \hat{s}^{2}+6 \hat{r}^{2} \hat{s}^{2}+\hat{r}^{4}\right.\right. \\
& \left.+\hat{s}^{4}\right) I_{1}(\hat{r} \hat{s})+128 \hat{r} \hat{s}\left(3-\hat{r}^{2}-\hat{s}^{2}\right) I_{0}(\hat{r} \hat{s})-\hat{r} \hat{s}(4 \\
& \left.\left.-\hat{r}^{2}\right)\left(4-\hat{s}^{2}\right)\left(32-4 \hat{r}^{2}-4 \hat{s}^{2}+\hat{r}^{2} \hat{s}^{2}\right)\right] / 512
\end{aligned}
$$

for $n=2$, and

$$
\begin{aligned}
W(r, s)= & e^{-\left(\hat{r}^{2}+\hat{s}^{2}\right) / 2}\left[1 1 5 2 \left(192-192 \hat{r}^{2}-192 \hat{s}^{2}+688 \hat{r}^{2} \hat{s}^{2}+96 \hat{r}^{4}+96 \hat{s}^{4}-272 \hat{r}^{4} \hat{s}^{2}-272 \hat{r}^{2} \hat{s}^{4}-16 \hat{r}^{6}-16 \hat{s}^{6}\right.\right. \\
& \left.+28 \hat{r}^{6} \hat{s}^{2}+28 \hat{r}^{2} \hat{s}^{6}+70 \hat{r}^{4} \hat{s}^{4}+\hat{r}^{8}+\hat{s}^{8}\right) I_{1}(\hat{r} \hat{s})+9216 \hat{r} \hat{s}\left(96-72 \hat{r}^{2}-72 \hat{s}^{2}+50 \hat{r}^{2} \hat{s}^{2}+15 \hat{r}^{4}+15 \hat{s}^{4}\right. \\
& \left.-7 \hat{r}^{4} \hat{s}^{2}-7 \hat{r}^{2} \hat{s}^{4}-\hat{r}^{6}-\hat{s}^{6}\right) I_{0}(\hat{r} \hat{s})-\hat{r} \hat{s}\left(24-12 \hat{r}^{2}+\hat{r}^{4}\right)\left(24-12 \hat{s}^{2}+\hat{s}^{4}\right) \\
& \left.\times\left(1728-480 \hat{r}^{2}-480 \hat{s}^{2}+192 \hat{r}^{2} \hat{s}^{2}+24 \hat{r}^{4}+24 \hat{s}^{4}-12 \hat{r}^{4} \hat{s}^{2}-12 \hat{r}^{2} \hat{s}^{4}+\hat{r}^{4} \hat{s}^{4}\right)\right] / 663552
\end{aligned}
$$

for $n=3$, where $I_{k}(x)$ is the modified Bessel function of order $k$ and we are using $\hat{r} \equiv r / l_{0}$ and $\hat{s} \equiv s / l_{0}$ for brevity. Even with analytic kernels, however, Eq. (36) cannot be handled within a finite numerical range without first analytically extracting the asymptotic [long-range (LR)] behavior of $u(r)$. For this purpose, we set $u^{\mathrm{LR}}(r)=2 / n r$, so that with $u(r)=u^{\mathrm{SR}}(r)+u^{\mathrm{LR}}(r)$, Eq. (36) becomes an equation for the short-range (SR) part,

$$
u^{\mathrm{SR}}(r)=v^{\mathrm{SR}}(r)+\frac{2 \pi \rho}{g(r)} \int_{0}^{\infty} d s s u^{\mathrm{SR}}(s) W(r, s),
$$

which contains only well-behaved, short-range functions. Here,

$$
v^{\mathrm{SR}}(r) \equiv v(r)-u^{\mathrm{LR}}(r)+\frac{2 \pi \rho}{g(r)} \int_{0}^{\infty} d s s u^{\mathrm{LR}}(s) W(r, s)
$$

must be evaluated analytically. We find $v^{\mathrm{SR}}(r)=0$ for $n=1$ [so that $u^{\mathrm{SR}}(r)=0, u(r)=2 / r$, and $\phi(r)=-2 \ln \left(r / l_{0}\right)$ is the anticipated exact solution for this case],

$$
v^{\mathrm{SR}}(r)=e^{-\hat{r}^{2} / 2} \hat{r}\left(32-14 \hat{r}^{2}+\hat{r}^{4}\right) / 32 g(r)
$$

for $n=2$, and

$$
\begin{aligned}
v^{\mathrm{SR}}(r)= & e^{-\hat{r}^{2} / 2} \hat{r}\left(1728-1440 \hat{r}^{2}+360 \hat{r}^{4}\right. \\
& \left.-34 \hat{r}^{6}+\hat{r}^{8}\right) / 864 g(r)
\end{aligned}
$$




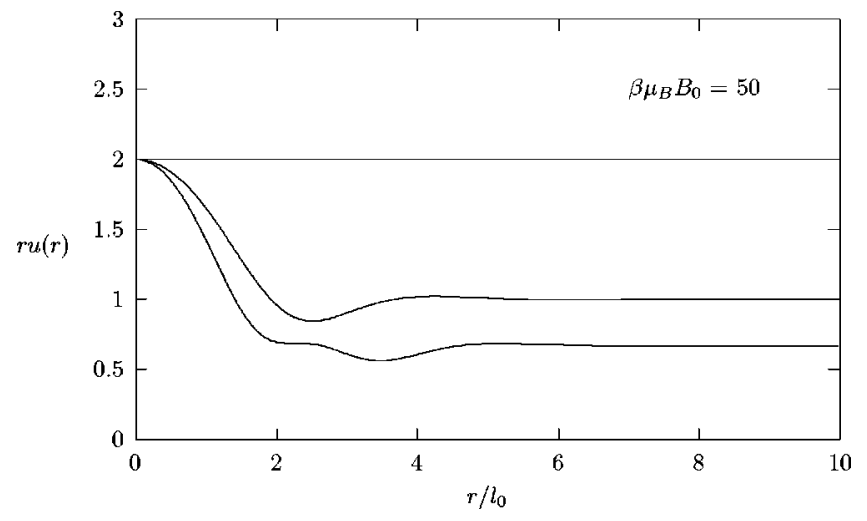

FIG. 8. Logarithmic derivative of the effective potentials, $r u(r)=-d \phi(r) / d \ln r$, obtained from the YBG equation for a twodimensional, noninteracting quantum electron gas in a magnetic field at a low temperature and reduced densities $2 \pi \rho l_{0}^{2}=1,2,3$, read from top to bottom.

for $n=3$. The computed results for these three cases are shown in Fig. 8 as $r u(r)$; for $n=1$, the solution is, of course, a constant, $r u(r)=2$, while for $n=2$ and $3, r u(r)$ makes a smooth transition from 2 at the origin to its longrange constant value of $2 / n$ over a distance $r \approx 5 l_{0}$, by which point particle pairs are essentially uncorrelated.

Evidently, equations for higher indices $n$ quickly become intricate to solve by this route, but these results are readily supplemented with the approximate HNC equation described in the following section. We show in Fig. 9 the effective potentials $\phi(r)$ obtained with both these methods, at $\beta \mu_{B} B_{0}=50$. Agreement between the approximate $\phi_{\mathrm{HNC}}(r)$ and the exact $\phi_{\mathrm{YBG}}(r)$ solutions for $n=1,2$, and 3 is reasonably good, especially at a large $r$.

Adapting to the present notation the total potential $V$ quoted by Jancovici ${ }^{8}$ for 2DOCP particles confined to a disk of radius $R$, we get a system potential, for large interparticle separations $r_{i j}$,

$$
\Phi_{N}^{(n)}\left(\boldsymbol{r}^{N}\right) \sim-\frac{2}{n} \sum_{i<j} \ln \left(\frac{r_{i j}}{l_{0}}\right)+\frac{1}{2} \sum_{i}\left(\frac{r_{i}}{l_{0}}\right)^{2}+\frac{N^{2}}{n}\left(\ln \frac{R}{l_{0}}-\frac{3}{4}\right),
$$

or, equivalently, a Boltzmann factor $W=\exp (-\Phi)$ given by

$$
W_{N}^{(n)}\left(\boldsymbol{r}^{N}\right) \sim \prod_{i<j}\left(\frac{r_{i j}}{l_{0}}\right)^{2 / n} \exp \left[-\frac{1}{2} \sum_{i}\left(\frac{r_{i}}{l_{0}}\right)^{2}\right],
$$

neglecting multiplicative constants, where $r_{i}$ is the radial distance from the disk center. The noninteracting electron gas in two dimensions is the basis for understanding the integral quantum Hall effect; ${ }^{2}$ we find that for the relevant incompressible states at low temperatures, this system is asymptotically described by the effective Boltzmann factor written above. For $n=1$, the Boltzmann factor is exact for all $r_{i j}$.

\section{HNC APPROACH TO THE EFFECTIVE POTENTIAL}

As noted earlier, the HNC integral equation of classical liquids has been used to model an effective potential for the free electron gas in three dimensions ${ }^{17}$ as well as in two. ${ }^{18,19}$
In this approach, one uses the equations ${ }^{9}$

$$
\begin{gathered}
\tilde{\gamma}(k)=\frac{\rho \widetilde{h}^{2}(k)}{1+\rho \widetilde{h}(k)}, \\
\phi(r)=\gamma(r)-\ln g(r)+B(r),
\end{gathered}
$$

which are exact, to first obtain the indirect correlation function $\gamma(r)$ from the known pair function $h(r)=g(r)-1$ and then to arrive at a solution for the effective potential $\phi(r)$ after an approximation is made for the so-called bridge function $B(r)$. In the HNC approximation, $B(r)$ is simply neglected, so that

$$
\phi_{\mathrm{HNC}}(r)=\gamma(r)-\ln g(r) .
$$

The calculation involves computing only transforms of pair functions and is noniterative. The HNC effective potentials obtained in this way at $\beta \mu_{B} B_{0}=1$ are graphically not very distinct from the YBG solutions of Fig. 6 and are not displayed.

As with the YBG calculation, numerical problems arise at low temperatures, where we have already found that the effective potential for the filled Landau levels evolves asymptotically into the 2DOCP interaction. Hansen and Levesque ${ }^{20}$ have reported numerical solutions of the HNC equation for the 2DOCP, specifically adapted to its long-range potential. We follow a similar procedure of extracting the divergence analytically with a long-range function

$$
\phi^{\mathrm{LR}}(r)=-\frac{1}{n}\left[2 \ln \left(\frac{r}{l_{0}}\right)+E_{1}\left(\frac{r^{2}}{2 l_{0}^{2}}\right)\right],
$$

whose dimensionless transform is

$$
\rho \widetilde{\phi}^{\mathrm{LR}}(k)=\frac{2}{\left(k l_{0}\right)^{2}} \exp \left[-\frac{1}{2}\left(k l_{0}\right)^{2}\right],
$$

so that numerical routines deal only with well-behaved functions. ${ }^{21}$ In Eq. (56), $E_{1}(x)$ is the exponential integral. Then, starting with the known function $h(r)=g(r)-1$, the steps of the solution are the following:

$$
\begin{aligned}
& \text { (1) } \tilde{h}(k)=2 \pi \int_{0}^{\infty} d r r h(r) J_{0}(k r), \\
& \text { (2) } \tilde{\gamma}^{\mathrm{SR}}(k)=\frac{\rho \widetilde{h}^{2}(k)}{1+\rho \widetilde{h}(k)}-\widetilde{\phi}^{\mathrm{LR}}(k), \\
& \text { (3) } \gamma^{\mathrm{SR}}(r)=\frac{1}{2 \pi} \int_{0}^{\infty} d k k \widetilde{\gamma}^{\mathrm{SR}}(k) J_{0}(k r), \\
& \text { (4) } \phi_{\mathrm{HNC}}(r)=\gamma^{\mathrm{SR}}(r)+\phi^{\mathrm{LR}}(r)-\ln g(r) .
\end{aligned}
$$

The numerical calculations now proceed smoothly. The HNC potentials at $\beta \mu_{B} B_{0}=50$ have already been displayed in Fig. 9, along with the YBG potentials from the preceding section. The deviations between the two for a small $r$ are a measure of the missing $B(r)$, a short-range function, in $\phi_{\mathrm{HNC}}(r)$. 


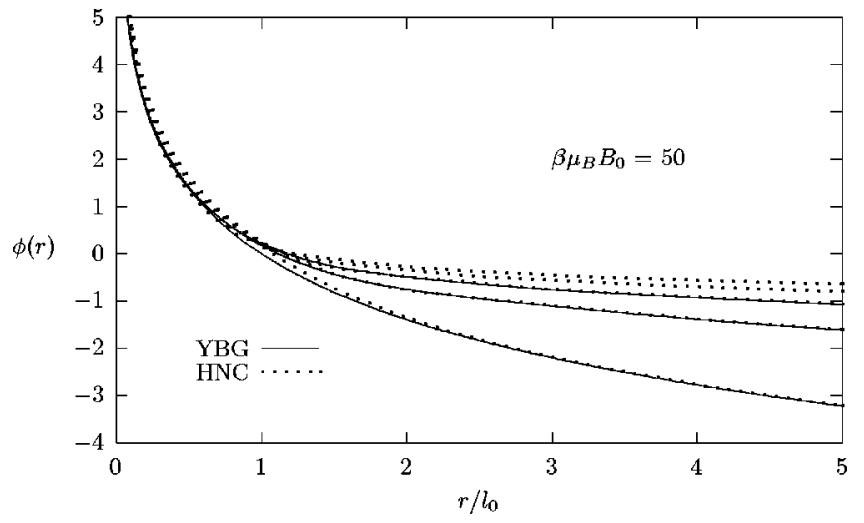

FIG. 9. Effective potentials for a two-dimensional, noninteracting quantum electron gas in a magnetic field at a low temperature, obtained from the YBG equation at reduced densities $2 \pi \rho l_{0}^{2}$ $=1,2,3$ and the $\mathrm{HNC}$ equation at reduced densities $2 \pi \rho l_{0}^{2}$ $=1,2,3,4,5$, read from bottom to top.

The Hankel transforms needed in these calculations are evaluated numerically. The discrete versions ${ }^{22}$ of the integrals (28) and (29) used in this work,

$$
\begin{aligned}
& \tilde{h}\left(k_{j}\right)=\frac{4 \pi}{K^{2}} \sum_{i=1}^{N_{r}-1} h\left(r_{i}\right) \frac{J_{0}\left(k_{j} r_{i}\right)}{J_{1}^{2}\left(K r_{i}\right)}, \\
& h\left(r_{i}\right)=\frac{1}{\pi R^{2}} \sum_{j=1}^{N_{r}-1} \tilde{h}\left(k_{j}\right) \frac{J_{0}\left(k_{j} r_{i}\right)}{J_{1}^{2}\left(k_{j} R\right)},
\end{aligned}
$$

preserve the orthogonality of the continuous Hankel transforms. In these expressions, $J_{n}(x)$ is the Bessel function of the order $n$ and the numerical grids are defined by $r_{i}$ $=\xi_{i} / K, k_{j}=\xi_{j} / R$, where $\xi_{j}$ is the $j$ th $\operatorname{root}$ of $J_{0}(x), K$ $\equiv k_{N_{r}}$, and $R \equiv r_{N_{r}}$; the range $R$ and number of grid points $N_{r}$ are free choices. In the present calculations, we have used $R / l_{0}=20$ and $N_{r}=1000$.

\section{CONCLUSIONS}

The electric potential due to an infinite line charge ${ }^{23}$ is, in Gaussian units, $v(r)=-2 \lambda \ln (r / L)$, where $\lambda$ is the linear charge density and $L$ is an arbitrary length. Then, with $L$ $=l_{0}$, the energy $w(r)$ associated with lengths $l_{0}$, containing charge $q=\lambda l_{0}$, of each of the two interacting infinite line charges is

$$
w(r)=-\left(2 q^{2} / l_{0}\right) \ln \left(r / l_{0}\right)=-\Gamma \ln \left(r / l_{0}\right),
$$

which is the defining potential of the $2 \mathrm{DOCP}^{7}$

In this work, we have found that the two-dimensional one-component plasma interaction also functions as an effective potential $\phi(r)$ for the simple quantum model of noninteracting electrons in a plane, subject to an external magnetic field, when they are taken to low temperatures and form incompressible states. The potential model is exact,

$$
\phi(r)=-2 \ln \left(r / l_{0}\right),
$$

for the filling factor $\nu=2 \pi \rho l_{0}^{2}=1$, and asymptotic,

$$
\phi(r) \sim-(2 / n) \ln \left(r / l_{0}\right),
$$

for filling factors $\nu=n>1$. The physical conditions $\beta \mu_{B} B_{0}$ $\gg 1$ and $2 \pi \rho l_{0}^{2}=n$ apply to the integral quantum Hall effect, and so we find that, for $\nu=1$, the noninteracting electron model for the integral quantum Hall effect maps into the classical two-dimensional one-component plasma through an effective Boltzman factor

$$
W_{N}^{(1)}\left(\boldsymbol{r}^{N}\right)=\prod_{i<j}\left(\frac{r_{i j}}{l_{0}}\right)^{2} \exp \left[-\frac{1}{2} \sum_{i}\left(\frac{r_{i}}{l_{0}}\right)^{2}\right] .
$$

It is, of course, well known that the 2DOCP has previously been invoked by Laughlin ${ }^{5,6}$ to construct an $N$-particle wave function $\psi_{m}$ that accounts for the principal features of the fractional quantum Hall effect. Expressed as an equivalent Boltzman factor $W=|\psi|^{2}$, Laughlin's solution is

$$
W_{N}^{(1 / m)}\left(\boldsymbol{r}^{N}\right)=\prod_{i<j}\left(\frac{r_{i j}}{l_{0}}\right)^{2 m} \exp \left[-\frac{1}{2} \sum_{i}\left(\frac{r_{i}}{l_{0}}\right)^{2}\right],
$$

with $m$ an odd integer, including $m=1$.

The coincidence of form in these effective Boltzmann factors for $\nu=n=1 / m=1$ in mathematical models for the integral and fractional quantum Hall effects is remarkable and nontrivial, since the two results arise from quite distinct origins.

(1) Laughlin presents Eq. (68) in the context of a system of electrons interacting through the Coulomb $e^{2} / r$ potential. Further, the result is constructed as the squared amplitude of a single ground-state wave function, which requires an explicit antisymmetrization, so that $m$ must be an odd integer in general.

(2) In this paper, Eq. (67) is obtained explicitly and exactly for a noninteracting system of electrons. Further, it is a sum over states, rather than the amplitude squared of a single wave function. The fact, that the quantum number $n=1$ is odd has here nothing to do with antisymmetrization; this property is built into the Slater sum from the very beginning, Eq. (2), and is reflected in its vanishing whenever two particles approach each other, which is the signature of "fermionicity."

One concludes that, at least for the filled lowest Landau level, $\nu=1$, Laughlin's variational wave function actually describes a noninteracting system. This is consistent with the fact that the electronic charge $e$ does not appear in these solutions unless multiplying the magnetic field, as $e B_{0}$, reflecting just the magnetic force on the electron.

\section{ACKNOWLEDGMENTS}

It is a pleasure to thank Professor George Parker for valuable discussions and an initiation into the ways of MATHEMATICA and Professor Charles Johnson for computer help over many years.

\section{APPENDIX: THE ONE-BODY SLATER SUM}

The vector potential in the Landau gauge is $\boldsymbol{A}=-B_{0} y \hat{\boldsymbol{l}}$, so the one-electron Hamiltonian becomes 


$$
\mathcal{H}_{1}=\frac{1}{2 m_{e}}\left[\left(i \hbar \frac{\partial}{\partial x}+e B_{0} y\right)^{2}+\left(i \hbar \frac{\partial}{\partial y}\right)^{2}\right] .
$$

The Schrödinger equation $\mathcal{H}_{1} \psi=\epsilon \psi$ is then solved by ${ }^{2}$

$$
\psi_{\kappa n}(x, y)=\frac{A_{n}}{L_{x}^{1 / 2}} \exp \left[i \kappa x-\frac{\left(y-\kappa l_{0}^{2}\right)^{2}}{2 l_{0}^{2}}\right] H_{n}\left(\frac{y-\kappa l_{0}^{2}}{l_{0}}\right),
$$

with $\kappa=2 \pi j / L_{x}$ for $j=0, \pm 1, \pm 2, \ldots, l_{0}=\left(\hbar / e B_{0}\right)^{1 / 2}$, and $A_{n}=\left(\pi l_{0}^{2}\right)^{-1 / 4}\left(2^{n} n !\right)^{-1 / 2}$. Further, $H_{n}(x)$ is the Hermite polynomial of the order $n$ and $L_{x}$ is the edge length of the rectangular area $A$ in the $x$ direction. The corresponding energy eigenvalues are $\epsilon_{n}=(2 n+1) \mu_{B} B_{0}, \quad n=0,1,2, \ldots$, where $\mu_{B}=e \hbar / 2 m_{e}$ is the Bohr magneton.

We seek to determine the one-body Slater sum

$$
\begin{aligned}
W_{1}(x, y)= & \Lambda^{2} \sum_{\kappa, n} e^{-\beta \epsilon_{n}} \psi_{\kappa n}^{*}(P x, P y) \psi_{\kappa n}(x, y)=\frac{\Lambda^{2}}{\left(\pi l_{0}^{2}\right)^{1 / 2} L_{x}} \\
& \times \sum_{\kappa} e^{i \kappa(x-P x)} e^{-\left(P y-\kappa l_{0}^{2}\right)^{2} / 2 l_{0}^{2}} e^{-\left(y-\kappa l_{0}^{2}\right)^{2} / 2 l_{0}^{2}} \\
& \times \sum_{n} \frac{e^{-(2 n+1) \beta \mu_{B} B_{0}}}{2^{n} n !} H_{n}\left(\frac{P y-\kappa l_{0}^{2}}{l_{0}}\right) H_{n}\left(\frac{y-\kappa l_{0}^{2}}{l_{0}}\right) .
\end{aligned}
$$

First we note that ${ }^{24}$

$$
\begin{aligned}
\sum_{n=0}^{\infty} & \frac{e^{-n \alpha}}{2^{n} n !} H_{n}(x) H_{n}(y) \\
& =\left(\frac{e^{\alpha}}{2 \sinh \alpha}\right)^{1 / 2} \exp \left[\frac{-\left(x^{2}+y^{2}\right) e^{-\alpha}+2 x y}{2 \sinh \alpha}\right]
\end{aligned}
$$

*Electronic address: fred_lado@ncsu.edu

${ }^{1}$ L.D. Landau, Z. Phys. 64, 629 (1930).

${ }^{2}$ T. Chakraborty and P. Pietiläinen, The Quantum Hall Effects (Springer-Verlag, Berlin, 1995).

${ }^{3}$ K. von Klitzing, G. Dorda, and M. Pepper, Phys. Rev. Lett. 45, 494 (1980).

${ }^{4}$ D.C. Tsui, H.L. Störmer, and A.C. Gossard, Phys. Rev. Lett. 48, 1559 (1982).

${ }^{5}$ R.B. Laughlin, Phys. Rev. Lett. 50, 1395 (1983).

${ }^{6}$ R.B. Laughlin, in The Quantum Hall Effect, edited by R.E. Prange and S.M. Girvin (Springer-Verlag, New York, 1990), p. 233.

${ }^{7}$ J.M. Caillol, D. Levesque, J.J. Weis, and J.P. Hansen, J. Stat. Phys. 28, 325 (1982).

${ }^{8}$ B. Jancovici, Phys. Rev. Lett. 46, 386 (1981).

${ }^{9}$ J.P. Hansen and I.R. McDonald, Theory of Simple Liquids (Academic, London, 1986).

${ }^{10}$ F. Lado, J. Chem. Phys. 47, 5369 (1967).

${ }^{11}$ O. Ciftja and S. Fantoni, Phys. Rev. B 56, 13290 (1997).

${ }^{12}$ O. Ciftja, Phys. Rev. B 59, 8132 (1999).
Using this in Eq. (A3) with $\alpha=2 \beta \mu_{B} B_{0}$ and simplifying, we arrive at

$$
\begin{aligned}
W_{1}(x, y)= & {\left[\Lambda^{2} /\left(2 \pi l_{0}^{2} \sinh \alpha\right)^{1 / 2} L_{x}\right] \sum_{\kappa} \exp [i \kappa(x-P x)} \\
& -(y-P y)^{2} / 2 l_{0}^{2} \tanh \alpha-\tanh (\alpha / 2)\left(y-\kappa l_{0}^{2}\right) \\
& \left.\times\left(P y-\kappa l_{0}^{2}\right) / l_{0}^{2}\right]
\end{aligned}
$$

The second sum, over $\kappa$, is effected as an integral. For a large $L_{x}, L_{x}^{-1} \Sigma_{\kappa} \rightarrow(2 \pi)^{-1} \int d \kappa$ and we get

$$
\begin{aligned}
& \frac{1}{2 \pi} \int_{-\infty}^{\infty} d \kappa \exp [i \kappa(x-P x)-\tanh (\alpha / 2) \\
& \left.\quad \times\left(y-\kappa l_{0}^{2}\right)\left(P y-\kappa l_{0}^{2}\right) / l_{0}^{2}\right] \\
& =\left[4 \pi l_{0}^{2} \tanh (\alpha / 2)\right]^{-1 / 2} \exp \left[-(x-P x)^{2} / 4 l_{0}^{2} \tanh (\alpha / 2)\right. \\
& \left.\quad+\tanh (\alpha / 2)(y-P y)^{2} / 4 l_{0}^{2}+i(x-P x)(y+P y) / 2 l_{0}^{2}\right],
\end{aligned}
$$

and so finally,

$$
\begin{aligned}
W_{1}(x, y)= & {\left[\Lambda^{2} / 4 \pi l_{0}^{2} \sinh (\alpha / 2)\right] } \\
& \times \exp \left[-(\boldsymbol{r}-P \boldsymbol{r})^{2} / 4 l_{0}^{2} \tanh (\alpha / 2)\right. \\
& \left.+i(x-P x)(y+P y) / 2 l_{0}^{2}\right]
\end{aligned}
$$

after some further simplification.
${ }^{13}$ R.K. Kamilla, J.K. Jain, and S.M. Girvin, Phys. Rev. B 56, 12411 (1997).

${ }^{14}$ G.E. Uhlenbeck and L. Gropper, Phys. Rev. 41, 79 (1932).

${ }^{15}$ K. Huang, Statistical Mechanics (Wiley, New York, 1987), Chap. 9.

${ }^{16}$ F.A. Stevens and M.A. Pokrant, J. Chem. Phys. 61, 3768 (1974).

${ }^{17}$ F.A. Stevens and M.A. Pokrant, Phys. Rev. A 8, 990 (1973).

${ }^{18}$ T. Chakraborty, Phys. Rev. B 57, 8812 (1998).

${ }^{19}$ O. Ciftja and S. Fantoni, Phys. Rev. B 58, 7898 (1998).

${ }^{20}$ J.P. Hansen and D. Levesque, J. Phys. C 14, L603 (1981).

${ }^{21}$ J.F. Springer, M.A. Pokrant, and F.A. Stevens, J. Chem. Phys. 58, 4863 (1973).

${ }^{22}$ F. Lado, J. Chem. Phys. 49, 3092 (1968); Comput. Phys. 8, 417 (1971).

${ }^{23}$ W.K.H. Panofsky and M. Phillips, Classical Electricity and Magnetism (Addison-Wesley, Reading, MA, 1962), Chap. 4.

${ }^{24}$ I.S. Gradshteyn and I.M. Ryzhik, Table of Integrals, Series, and Products (Academic Press, New York, 1980). Integral 7.374.10 and the orthogonality of the Hermite polynomials yield this result. 